\title{
On the 1-nucleolus
}

\author{
A. Estévez-Fernández ${ }^{1}$ (D) P. Borm² ${ }^{2}$ - M. G. Fiestras-Janeiro ${ }^{3}$. \\ M. A. Mosquera ${ }^{3}$. E. Sánchez-Rodríguez ${ }^{3}$
}

\begin{abstract}
This paper analyzes the 1-nucleolus and, in particular, its relation to the nucleolus. It is seen that, contrary to the nucleolus, the 1-nucleolus can be computed in polynomial time due to a characterization using a combination of standard bankruptcy rules for associated bankruptcy problems. Sufficient conditions on a compromise stable game are derived such that the 1-nucleolus and the nucleolus coincide.
\end{abstract}

Keywords 1-nucleolus - Compromise stable games · Aumann-Maschler rule · Nucleolus

\section{Introduction}

Cooperative transferable utility games (TU-games) have proven effective to analyze problems where the joint profits obtained by a joint collaboration have to be shared among the individuals involved (the grand coalition). In order to decide on a "fair" or "just" distribution of the joint profits (a solution), benchmarks are used: the joint profits that any subgroup of individuals (a coalition) could obtain by cooperation without any help from the other members of the grand coalition that are outside this

A. Estévez-Fernández

arantza.estevezfernandez@vu.nl

1 Tinbergen Institute and Department of Econometrics and Operations Research, Vrije Universiteit Amsterdam, Amsterdam, The Netherlands

2 Center and Department of Econometrics and Operations Research, Tilburg University, Tilburg, The Netherlands

3 Department of Statistics and Operations Research, Universidade de Vigo, Vigo, Spain 
subgroup. This means that the description of a cooperative game in general requires the computation of $2^{n}$ values, with $n$ being the number of members of the grand coalition. For this reason, the computation of solutions that are based on all coalitional values is NP-hard.

In a general framework, there are several solutions to TU-games available that, in principle, need as input all coalitional values. Among the most studied are the core (Gillies 1953), the nucleolus (Schmeidler 1969), the Shapley value (Shapley 1953) and the compromise value (Tijs 1981).

This paper analyzes the possibility of reducing the number of input variables with respect to the nucleolus of a TU-game by studying the 1-nucleolus, a member of the class of generalized nucleoli introduced in Maschler et al. (1992) and conceptually related to the notion of the $k$-core cover as studied in Sánchez-Rodríguez et al. (2015). For TU-games with a nonempty imputation set, the nucleolus is a solution based on the idea that a fair distribution of the total worth should (lexicographically) minimize the sorted vector of the excesses (or complaints) associated with all possible coalitions. Given an imputation $x$ and a coalition $S$, the excess measures the dissatisfaction of $S$ at $x$. There are different algorithms to compute the nucleolus, see the Kopelowitz algorithm (Kopelowitz 1967) or the Maschler-Peleg-Shapley algorithm (Maschler et al. 1979). The complexity of these algorithms, however, is exponential in the number of players, and therefore useful only for relatively small games. Still, there are classes of games, such as assignment games (Shapley and Shubik 1972), where the complexity of these algorithms only grows polynomically in the number of players. That fact has allowed to develop special algorithms to obtain the nucleolus when the game has a special underlying structure. Nevertheless, in most applications where many players are involved, the task of computing the nucleolus can be very difficult.

This paper focuses on the 1-nucleolus which is based on computing the excesses only of coalitions of size 1 and $n-1$. By reducing the number of variables involved in the computation of the 1-nucleolus with respect to the nucleolus, we also decrease its computational difficulty. We characterize the 1-nucleolus using a combination of standard bankruptcy rules of an associated bankruptcy problem, the computations of which are done in polynomial time. Next, we analyze under which conditions the nucleolus and 1-nucleolus of compromise stable games coincide. Finally, without aiming for a full characterization of the 1-nucleolus, we study several properties on the class of TU-games with a nonempty core and use them to compare the 1-nucleolus and the nucleolus. In particular, it is seen that the 1-nucleolus satisfies individual superadditivity gains to the grand coalition and first agent consistency, which are not satisfied by the nucleolus. Unfortunately, just like the nucleolus, the 1-nucleolus does not satisfy aggregate monotonicity either.

The outline of the paper is as follows. Section 2 recalls basic concepts and results that will be used throughout the paper. Section 3 formally defines the 1-nucleoli. In Sect. 4, we characterize the 1-nucleolus by means of a combination of bankruptcy rules. Section 5 analyzes the 1-nucleolus in relation with the nucleolus for compromise stable games and Sect. 6 is dedicated to open questions and to comparing the 1-nucleolus and nucleolus based on some desirable properties. 


\section{Preliminaries}

In this section, we survey some well-known concepts and results that will be used in the subsequent sections.

For $x, y \in \mathbb{R}^{n}$, we say that $x$ is lexicographically smaller than $y, x<_{L} y$, if there is $m \in\{1, \ldots, n\}$ such that $x_{l}=y_{l}$ for every $1 \leq l<m$ and $x_{m}<y_{m}$. Moreover, $x \leq_{L} y$ if either $x=y$, or $x<_{L} y$.

A transferable utility game (TU-game) is a pair $(N, v)$ where $N$ is a finite set of players and $v: 2^{N} \rightarrow \mathbb{R}$ satisfies $v(\emptyset)=0$, where $2^{N}$ denotes the set of subsets or coalitions of $N$. In general, $v(S)$ represents the value of coalition $S$, that is, the joint payoff that can be obtained by this coalition when its members decide to cooperate. Let $G^{N}$ be the set of all TU-games with player set $N$. Given $S \subseteq N$, let $|S|$ be the number of players in $S$.

The main focus within a cooperative setting is on how to share the total joint payoff obtained when all players decide to cooperate. Given a TU-game $v \in G^{N}$, the imputation set of $v, I(v)$, is the set of efficient allocations that are individually rational. Formally,

$$
I(v)=\left\{x \in \mathbb{R}^{N} \| \sum_{i \in N} x_{i}=v(N), x_{i} \geq v(\{i\}) \text { for all } i \in N\right\} .
$$

Note that the imputation set is nonempty if, and only if,

$$
\sum_{i \in N} v(\{i\}) \leq v(N)
$$

We denote by $I^{N}$ the set of all TU-games with player set $N$ and nonempty imputation set.

The core of $v \in G^{N}, \mathcal{C o r e}(v)$, was first introduced in Gillies (1953) and is defined as the set of efficient allocations that are stable, in the sense that no coalition has an incentive to deviate. Formally,

$$
\operatorname{Core}(v)=\left\{x \in \mathbb{R}^{N} \mid \sum_{i \in N} x_{i}=v(N), \sum_{i \in S} x_{i} \geq v(S) \text { for all } S \subseteq N\right\}
$$

Bondareva (1963) and Shapley (1967) established that a game $v \in G^{N}$ has a nonempty core if, and only if, it is balanced. Before introducing balanced games, we need to fix some notation.

Let $\emptyset \neq S \subseteq N$ and let $e^{S} \in \mathbb{R}^{N}$ be the characteristic vector of $S$, defined as $e_{i}^{S}=1$ if $i \in S$ and $e_{i}^{S}=0$ if $i \notin S$. A family $\mathcal{B}$ of nonempty subcoalitions of $S$ is called balanced on $S$ if there are positive weights $\delta=\left\{\delta_{T}\right\}_{T \in \mathcal{B}}, \delta_{T}>0$ for all $T \in \mathcal{B}$, such that $\sum_{T \in \mathcal{B}} \delta_{T} e^{T}=e^{S}$ or, equivalently, $\sum_{T \in \mathcal{B}: T \ni i} \delta_{T}=1$ for all $i \in S$ and $\sum_{T \in \mathcal{B}: T \ni i} \delta_{T}=0$ for all $i \in N \backslash S$. We denote by $\mathcal{F}(S)$ the set of balanced families of $S$. Given a balanced family $\mathcal{B}$, we denote by $\Delta(\mathcal{B})$ the set of positive weights satisfying 
the balancedness condition. A game $v \in G^{N}$ is called balanced if for all balanced families $\mathcal{B} \in \mathcal{F}(N)$ and all $\left\{\delta_{S}\right\}_{S \in \mathcal{B}} \in \Delta(\mathcal{B}), \sum_{S \in \mathcal{B}} \delta_{S} v(S) \leq v(N)$.

A well-established one-point solution concept is the nucleolus, introduced in Schmeidler (1969). Let $v \in I^{N}$ and let $x \in I(v)$. We denote the excess of coalition $S \in 2^{N}$ with respect to $x$ by

$$
e(S, x)=v(S)-\sum_{j \in S} x_{j} .
$$

Moreover, we denote by $\theta(x) \in \mathbb{R}^{2^{|N|}}$ the vector whose coordinates are the excesses $e(S, x)$ arranged in non-increasing order, that is, $\theta_{l}(x) \geq \theta_{m}(x)$ for every $1 \leq l \leq$ $m \leq 2^{|N|}$. The nucleolus of $v \in G^{N}, \operatorname{nuc}(v)$, is defined as

$$
n u c(v)=\left\{x \in I(v) \mid \theta(x) \leq_{L} \theta(y) \text { for all } y \in I(v)\right\}
$$

Schmeidler (1969) showed that the nucleolus of a game with a nonempty imputation set exists and is unique. The nucleolus is invariant with respect to positive affine transformations, i.e. for $v \in I^{N}, \alpha>0$, and $a \in \mathbb{R}^{N}$, it follows $n u c(\alpha v+a)=$ $\alpha n u c(v)+a$ with $(\alpha v+a)(S)=\alpha v(S)+\sum_{j \in S} a_{j}$ for every $S \in 2^{N}$.

Tijs and Lipperts (1982) introduced the core cover. Let $v \in I^{N}$ and $i \in N$. The utopia value of player $i, M_{i}(v)$, is defined as

$$
M_{i}(v)=v(N)-v(N \backslash\{i\}) .
$$

The minimal right of player $i, m_{i}(v)$, is defined as

$$
m_{i}(v)=\max _{S \subseteq N \backslash\{i\}}\left\{v(S \cup\{i\})-\sum_{j \in S} M_{j}(v)\right\} .
$$

Note that, in general, we need $2^{|N|-1}$ values to compute the minimal right of a player. Thus, in general, the computation of minimal rights is NP-hard. The utopia vector is given by $M(v)=\left(M_{i}(v)\right)_{i \in N}$ and the minimal right vector is given by $m(v)=$ $\left(m_{i}(v)\right)_{i \in N}$. The core cover of $v \in G^{N}, \mathcal{C C}(v)$, is defined as

$$
\mathcal{C C}(v)=\{x \in I(v) \mid m(v) \leq x \leq M(v)\} .
$$

It can be verified that $\mathcal{C}$ ore $(v) \subseteq \mathcal{C C}(v) \subseteq I(v)$. A TU game $v \in I^{N}$ is compromise admissible if $\mathcal{C C}(v) \neq \emptyset$. A compromise admissible game is compromise stable if Core $(v)=\mathcal{C C}(v)$. Quant et al. (2005) characterized the family of compromise stable games.

Theorem 2.1 (Quant et al. 2005). Let $v \in I^{N}$ be compromise admissible. Then, $v$ is compromise stable if, and only if, $v(S) \leq \max \left\{\sum_{j \in S} m_{j}(v), v(N)-\sum_{j \in N \backslash S} M_{j}(v)\right\}$ for every $S \subseteq N$. 
An important subclass of balanced games is the class of convex games, as introduced in Shapley (1971). A game $v \in G^{N}$ is convex if $v(S \cup\{i\})-v(S) \leq v(T \cup\{i\})-v(T)$ for every $i \in N$ and $S \subseteq T \subseteq N \backslash\{i\}$.

A bankruptcy problem is described by $(N, E, c)$, with $N$ a finite set of players, $E>0$, and $c \in \mathbb{R}^{N}$ such that $c_{i} \geq 0$ for all $i \in N$ and $\sum_{i \in N} c_{i} \geq E$. O'Neill (1982) defines the bankruptcy game associated to a bankruptcy problem $(N, E, c)$, as

$$
v_{E, c}(S)=\max \left\{0, E-\sum_{i \in N \backslash S} c_{i}\right\} \text { for every } S \in 2^{N} .
$$

In fact, Quant et al. (2005) show that a game is convex and compromise stable game if, and only if, it is $S$-equivalent ${ }^{1}$ to a bankruptcy game. Aumann and Maschler (1985) show that the nucleolus of a bankruptcy game corresponds to the Aumann-Maschler rule of the corresponding bankruptcy problem.

\section{1-nucleolus}

This section focuses on the 1-nucleolus of a game by considering excesses only of coalitions of size at most 1 and at least $|N|-1$. In order to formally define the 1nucleolus, we need to fix some notation. Let $N$ be a finite set, we denote

$$
\mathcal{C}^{1}(N)=\left\{S \in 2^{N}|| S \mid \leq 1 \text { or }|S| \geq|N|-1\right\} .
$$

If no confusion arises, we write $\mathcal{C}^{1}$ instead of $\mathcal{C}^{1}(N)$. Given $v \in I^{N}$ and $x \in I(v)$, we write $\theta^{1}(x) \in \mathbb{R}^{2(|N|+1)}$ the vector whose coordinates are the excesses $e(S, x)$, with $S \in \mathcal{C}^{1}$, arranged in non-increasing order, that is, $\theta_{l}^{1}(x) \geq \theta_{m}^{1}(x)$ for every $1 \leq l \leq m \leq 2(|N|+1)$.

Definition 3.1 Let $v \in I^{N}$. The 1-nucleolus is defined by

$$
n u c^{1}(v)=\left\{x \in I(v) \mid \theta^{1}(x) \leq_{L} \theta^{1}(y) \text { for all } y \in I(v)\right\} .
$$

Note that for $|N|=3, \mathcal{C}^{1}(N)=2^{N}$. As a consequence, the 1 -nucleolus and nucleolus of 3-players games coincide. Moreover, just like the nucleolus, the 1-nucleolus is invariant with respect to positive affine transformations.

Theorem 3.2 (cf. Schmeidler 1969) Let $v \in I^{N}$. Then, nuc ${ }^{1}(v)$ exists and is unique.

The characterization of the nucleolus in Kohlberg (1971) can be translated to the 1-nucleolus as already pointed out in Maschler et al. (1992). For this, we need to obtain a partition of the coalitions in $N$ of size at most 1 or at least $|N|-1$. Let $v \in I^{N}$ and let $x \in I(v)$. Let $B_{0}^{1}(x, v)=\left\{\{i\} \subseteq N \mid x_{i}=v(\{i\})\right\}$ and define recursively

\footnotetext{
${ }^{1}$ Two games $v, w \in G^{N}$ are $S$-equivalent if there exists $\alpha>0$ and $a \in \mathbb{R}^{N}$ such that $v(S)=\alpha w(S)+$ $\sum_{i \in S} a_{i}$ for every $S \subseteq N$.
} 


$$
\begin{aligned}
B_{l}^{1}(x, v)= & \left\{S \in \mathcal{C}^{1}(N) \backslash\left(\cup_{m=1}^{l-1} B_{m}^{1}(x, v)\right) \mid e(S, x) \geq e(R, x)\right. \\
& \text { for every } \left.R \in \mathcal{C}^{1}(N) \backslash\left(\cup_{m=1}^{l-1} B_{m}^{1}(x, v)\right)\right\}
\end{aligned}
$$

for $l \in\{1, \ldots, p\}$, with $p$ such that $B_{p}^{1}(x, v) \neq \varnothing$ and $\left\langle B_{1}^{1}(x, v), \ldots, B_{p}^{1}(x, v)\right\rangle$ forms a partition of the set of coalitions of $\mathcal{C}^{1}(N)$. For $l \in\{1, \ldots, p\}$, let $\mathcal{B}^{1, l}(x, v)=$ $\cup_{m=1}^{l} B_{m}^{1}(x, v)$.

Theorem 3.3 (cf. Kohlberg 1971; Maschler et al. 1992). Let $v \in I^{N}$. Then, $x$ is the 1-nucleolus of $v$ if, and only if, for every $l \in\{1, \ldots, p\}$, there exists $B_{0}^{1, l}(x, v) \subseteq$ $B_{0}^{1}(x, v)$ such that $B_{0}^{1, l}(x, v) \cup \mathcal{B}^{1, l}(x, v)$ is balanced.

\section{1-nucleolus and bankruptcy}

The 1-nucleolus only takes into account the information provided by the value of the singletons (individual coalitions), the value of the $|N|-1$ player coalitions, and the value of the grand coalition. Thus, the information needed stems from $2|N|+1$ coalitions.

This section shows that the 1-nucleolus is related to the Aumann-Maschler rule of bankruptcy problems (see Aumann and Maschler 1985), the constrained equal losses rule for bankruptcy problems, and the equal share rule. Moreover, the 1-nucleolus of a balanced game can be described as the Aumann-Maschler rule of an associated bankruptcy problem. As a consequence, it turns out that the 1-nucleolus and the nucleolus of bankruptcy games coincide (see O'Neill 1982; Aumann and Maschler 1985; Quant et al. 2005). We recall some well-known bankruptcy rules in the literature. The equal share rule of a bankruptcy problem $(N, E, c), \mathrm{ES}(N, E, c)$, assigns

$$
\mathrm{ES}_{j}(N, E, c)=\frac{E}{|N|} \text { for every } j \in N
$$

The constrained equal awards rule of a bankruptcy problem $(N, E, c), \operatorname{CEA}(N, E, c)$, assigns

$$
\mathrm{CEA}_{j}(N, E, c)=\min \left\{\lambda, c_{j}\right\} \text { to every } j \in N,
$$

with $\lambda \in \mathbb{R}_{+}$chosen such that $\sum_{j \in N} \operatorname{CEA}_{j}(N, E, c)=E$. The Aumann-Maschler rule of a bankruptcy problem $(N, E, c), \operatorname{AM}(N, E, c)$, is given by

$$
\operatorname{AM}(N, E, c)= \begin{cases}\operatorname{CEA}\left(N, E, \frac{1}{2} c\right) & \text { if } E \leq \frac{1}{2} \sum_{j \in N} c_{j} \\ c-\operatorname{CEA}\left(N, \sum_{j \in N} c_{j}-E, \frac{1}{2} c\right) & \text { otherwise }\end{cases}
$$

Aumann and Maschler (1985) showed that the Aumann-Maschler rule of a bankruptcy problem corresponds to the nucleolus of the associated bankruptcy game. 
To conclude, the constrained equal losses rule of a bankruptcy problem $(N, E, c)$, $\operatorname{CEL}(N, E, c)$, is defined as

$$
\operatorname{CEL}_{j}(N, E, c)=\max \left\{0, c_{j}-\lambda\right\} \text { for every } j \in N,
$$

where $\lambda$ is chosen such that $\sum_{j \in N} \operatorname{CEL}_{j}(N, E, c)=E$.

For $v \in I^{N}$, we define the zero-normalization of $v, v_{0} \in I^{N}$, as

$$
v_{0}(S)=v(S)-\sum_{j \in S} v(\{j\}) \text { for every } S \in 2^{N} .
$$

Note that $n u c^{1}\left(v_{0}\right)=n u c^{1}(v)-(v(\{j\}))_{j \in N}$. Therefore, when describing the 1-nucleolus, we can assume that $v=v_{0}$, that is, that $v$ is zero-normalized.

The following result fully describes the 1-nucleolus by means of a combination of standard bankruptcy solutions to associated bankruptcy problems.

Theorem 4.1 Let $v \in I^{N}$ with $v=v_{0}$. Let $E=v(N)$ and let $c \in \mathbb{R}^{N}$ be defined by $c_{j}=v(N)-v(N \backslash\{j\})$ for every $j \in N$.

(i) If $c_{j} \geq 0$ for every $j \in N$, then,

$$
n u c^{1}(v)= \begin{cases}A M(N, E, c) & \text { if } E \leq \sum_{j \in N} c_{j} \\ c+E S\left(N, E-\sum_{j \in N} c_{j}, c\right) & \text { if } E>\sum_{j \in N} c_{j}\end{cases}
$$

(ii) If $c_{j}<0$ for some $j \in N$, let $c^{+} \in \mathbb{R}^{N}$ be defined by $c_{j}^{+}=\max \left\{0, c_{j}\right\}$ for every $j \in N$ and let $c^{\min } \in \mathbb{R}^{N}$ be defined as $c_{j}^{\min }=c_{j}-\min \left\{c_{l} \mid l \in N\right\}$ for every $j \in N$. Then,

$$
n u c^{1}(v)= \begin{cases}A M\left(N, E, c^{+}\right) & \text {if } E \leq \sum_{j \in N} c_{j}^{+}, \\ C E L\left(N, E, c^{\min }\right) & \text { if } \sum_{j \in N} c_{j}^{+}<E \leq \sum_{j \in N} c_{j}^{\min } \\ c+E S\left(N, E-\sum_{j \in N} c_{j}, c\right) & \text { if } E>\sum_{j \in N} c_{j}^{\min }\end{cases}
$$

Proof See "Appendix".

Since all rules used in Theorem 4.1 are computed in polynomial time and only the values of coalitions of size $1,|N|-1$, and $|N|$ are used, the 1-nucleolus is also computed in polynomial time. The next result provides an explicit connection of the 1-nucleolus for balanced games to the Aumann-Maschler rule.

Theorem 4.2 Let $v \in I^{N}$ be a balanced game with $v=v_{0}$. Then,

$$
n u c^{1}(v)=A M(N, E, c)
$$

with $E=v(N)$ and $c \in \mathbb{R}^{N}$ defined as $c_{j}=v(N)-v(N \backslash\{j\})$ for every $j \in N$. 
Proof By Theorem 4.1 (i), it suffices to show that $c_{j} \geq 0$ for every $j \in N$ and that $E \leq \sum_{j \in N} c_{j}$

Let $j \in N$. Since $v$ is balanced, we have that $v(N) \geq v(\{j\})+v(N \backslash\{j\})=$ $v(N \backslash\{j\})$. Therefore, $c_{j}=v(N)-v(N \backslash\{j\}) \geq 0$. fore,

Moreover, since $v$ is balanced, we have that $\sum_{j \in N} \frac{1}{|N|-1} v(N \backslash\{j\}) \leq v(N)$. There-

$$
\begin{aligned}
E & =v(N)=v(N)+(|N|-1) \sum_{j \in N} \frac{1}{|N|-1} v(N \backslash\{j\})-\sum_{j \in N} v(N \backslash\{j\}) \\
& \leq v(N)+(|N|-1) v(N)-\sum_{j \in N} v(N \backslash\{j\})=\sum_{j \in N}(v(N)-v(N \backslash\{j\}))=\sum_{j \in N} c_{j} .
\end{aligned}
$$

As a consequence, we have

Theorem 4.3 Let $(N, E, c)$ be a bankruptcy problem and let $\left(N, v_{E, c}\right)$ be the corresponding bankruptcy game. Then, nuc $\left(v_{E, c}\right)=n u c^{1}\left(v_{E, c}\right)$.

Proof Let $w \in G^{N}$ be the zero-normalization of $v_{E, c}$, that is $w(S)=v_{E, c}(S)-$ $\sum_{j \in S} v_{E, c}(\{j\})$ for all $S \in 2^{N}$. Then,

$$
\begin{aligned}
n u c^{1}\left(v_{E, c}\right)= & \left(v_{E, c}(\{j\})\right)_{j \in N}+n u c^{1}(w) \\
= & \left(v_{E, c}(\{j\})\right)_{j \in N}+\operatorname{AM}\left(N, w(N),(w(N)-w(N \backslash\{j\}))_{j \in N}\right) \\
= & \left(v_{E, c}(\{j\})\right)_{j \in N} \\
& +\operatorname{AM}\left(N, v_{E, c}(N)-\sum_{j \in N} v_{E, c}(\{j\}), M\left(v_{E, c}\right)-\left(v_{E, c}(\{j\})\right)_{j \in N}\right) \\
= & m\left(v_{E, c}\right)+\operatorname{AM}\left(N, v_{E, c}(N)-\sum_{j \in N} m_{j}\left(v_{E, c}\right), M\left(v_{E, c}\right)-m\left(v_{E, c}\right)\right) \\
= & \operatorname{AM}\left(N, v_{E, c}(N), M\left(v_{E, c}\right)\right) \\
= & n u c\left(v_{E, c}\right)
\end{aligned}
$$

where the third equality follows from $M_{i}(w)=w(N)-w(N \backslash\{i\})=M_{i}\left(v_{E, c}\right)-$ $v_{E, c}(\{i\})$ for every $i \in N$, the fourth equality is a direct consequence of $v_{E, c}(\{j\})=$ $m_{j}\left(v_{E, c}\right)$ for every $j \in N$, and the fifth equality follows from the fact that the AumannMaschler rule satisfies the property of minimal rights first (see Thomson 2003).

As a consequence of Theorem 4.3, the nucleolus and 1-nucleolus of convex and compromise stable games coincide since every convex and compromise stable game is $S$-equivalent to a bankruptcy game (cf. Quant et al. 2005).

\section{1-nucleolus and nucleolus}

Quant et al. (2005) characterize the nucleolus of compromise stable games. 
Theorem 5.1 (Quant et al. 2005). Let $v \in I^{N}$ be a compromise stable game. Then,

$$
n u c(v)=m(v)+A M\left(N, v(N)-\sum_{j \in N} m_{j}(v), M(v)-m(v)\right) .
$$

By Theorem 5.1, the nucleolus of a compromise stable game depends on the minimal right vector of the game and its computation is, therefore, still NP-hard. The following example shows that the 1-nucleolus might not belong to the core cover of a compromise stable game. Furthermore, it illustrates that the 1-nucleolus and the nucleolus of such a game need not coincide.

Example 5.2 Consider $v \in I^{N}$ with $N=\{1,2,3,4\}$,

$$
\begin{aligned}
& v(\{1\})=0, v(\{2\})=0, v(\{3\})=0, v(\{4\})=0, \\
& v(\{1,2\})=0, v(\{1,3\})=0, v(\{1,4\})=1, v(\{2,3\})=3, \\
& \quad v(\{2,4\})=0, v(\{3,4\})=4, \\
& v(\{1,2,3\})=0, v(\{1,2,4\})=1, v(\{1,3,4\})=5, v(\{2,3,4\})=5, v(N)=5 .
\end{aligned}
$$

Here, $m(v)=(0,0,3,1)$ and $M(v)=(0,0,4,5)$. One readily verifies (using Theorem 2.1) that $v$ is compromise stable. Using Theorem 5.1, we have

$$
n u c(v)=(0,0,3,1)+A M(N, 1,(0,0,1,4))=(0,0,3.5,1.5) \in \mathcal{C C}(v)
$$

However, using Theorem 4.1, we have

$$
n u c^{1}(v)=A M(N, 5,(0,0,4,5))=(0,0,2,3) \notin \mathcal{C C}(v) .
$$

Notice that in the example above, both the nucleolus and the 1-nucleolus are obtained through the Aumann-Maschler rule, but they provide different allocations. This difference arises from the fact that we first allocate the minimal rights in the nucleolus and then we apply the Aumann-Maschler rule, while in the case of the 1-nucleolus we first allocate the vector $(v(\{i\}))_{i \in N}$ and then we apply the Aumann-Maschler rule. Thus, some of the coordinates of the 1-nucleolus may be smaller than the corresponding coordinates of the minimal rights vector. Precisely that difference makes the 1-nucleolus of a compromise stable game easier to compute than the nucleolus, since one does not need the minimal rights vector. Next, we provide some conditions for the nucleolus and 1-nucleolus of a compromise stable game to coincide.

Theorem 5.3 Let $v \in I^{N}$ be compromise stable. Let $E=v(N)-\sum_{j \in N} v(\{j\})$ and $c_{j}=M_{j}(v)-v(\{j\})$ for every $j \in N$.

(i) If $m_{j}\left(v_{E, c}\right)=m_{j}(v)-v(\{j\})$ for every $j \in N$, then, $n u c^{1}(v)=n u c(v)$.

(ii) If $m_{j}(v)=\max \left\{v(\{j\}), v(N)-\sum_{k \in N \backslash\{j\}} M_{k}(v)\right\}$ for every $j \in N$, then, $n u c^{1}(v)=n u c(v)$.

(iii) If either $m(v)<M(v)$, or $m(v)=M(v)$, then, $n u c^{1}(v)=n u c(v)$. 
Proof We assume, without loss of generality, that $v=v_{0}$. Note that $E=v(N)-$ $\sum_{j \in N} v(\{j\})=v(N)$ and $c_{j}=M_{j}(v)-v(\{j\})=M_{j}(v)$ for every $j \in N$. Since $v$ is compromise stable, $v$ is balanced and, therefore, $n u c^{1}(v)=\operatorname{AM}(N, E, c)$ by Theorem 4.2.

(i) Let $m_{j}\left(v_{E, c}\right)=m_{j}(v)-v(\{j\})=m_{j}(v)$ for every $j \in N$. Then,

$$
\begin{aligned}
n u c^{1}(v) & =\operatorname{AM}(N, E, c) \\
& =m\left(v_{E, c}\right)+\operatorname{AM}\left(N, E-\sum_{j \in N} m_{j}\left(v_{E, c}\right), c-m\left(v_{E, c}\right)\right) \\
& =m(v)+\operatorname{AM}\left(N, v(N)-\sum_{j \in N} m_{j}(v), M(v)-m(v)\right) \\
& =n u c(v)
\end{aligned}
$$

where the second equality follows from the fact that the Aumann-Maschler rule satisfies minimal rights first (see Thomson 2003), the third one is a direct consequence of $m_{j}\left(v_{E, c}\right)=m_{j}(v)-v(\{j\})=m_{j}(v)$ for every $j \in N$, and the last one follows from Theorem 5.1.

(ii) Let $m_{j}(v)=\max \left\{v(\{j\}), v(N)-\sum_{k \in N \backslash\{j\}} M_{k}(v)\right\}$ for every $j \in N$. We show that $m_{j}\left(v_{E, c}\right)=m_{j}(v)-v(\{j\})=m_{j}(v)$ for every $j \in N$.

Since $\left(N, v_{E, c}\right)$ is convex, we have $m_{j}\left(v_{E, c}\right)=v_{E, c}(\{j\})$ for every $j \in N$. Then, for $j \in N$,

$$
\begin{aligned}
m_{j}\left(v_{E, c}\right) & =v_{E, c}(\{j\}) \\
& =\max \left\{0, E-\sum_{k \in N \backslash\{j\}} c_{k}\right\} \\
& =\max \left\{0, v(N)-\sum_{k \in N} v(\{k\})-\sum_{k \in N \backslash\{j\}}\left(M_{k}(v)-v(\{k\})\right)\right\} \\
& =\max \left\{0, v(N)-\sum_{k \in N \backslash\{j\}} M_{k}(v)\right\} \\
& =\max \left\{v(\{j\}), v(N)-\sum_{k \in N \backslash\{j\}} M_{k}(v)\right\} \\
& =m_{j}(v)-v(\{j\})
\end{aligned}
$$

Then, by (i), we have that $n u c^{1}(v)=n u c(v)$.

(iii) First, let $m(v)<M(v)$. We show that $m_{j}(v)=\max \{v(\{j\}), v(N)-$ $\left.\sum_{k \in N \backslash\{j\}} M_{k}(v)\right\}$ for every $j \in N$. By (ii), we then have that $n u c^{1}(v)=n u c(v)$. On the contrary, suppose that there exists $i \in N$ and $S \in 2^{N} \backslash\{\emptyset, N\}$ with $S \ni i$ such that $m_{i}(v)=v(S)-\sum_{k \in S \backslash\{i\}} M_{k}(v)>\max \{v(\{i\}), v(N)-$ $\left.\sum_{k \in N \backslash\{i\}} M_{k}(v)\right\}$. Then, we arrive at a contradiction since 


$$
\begin{aligned}
m_{i}(v) & =v(S)-\sum_{k \in S \backslash\{i\}} M_{k}(v) \\
& \leq \max \left\{\sum_{k \in S} m_{k}(v), v(N)-\sum_{k \in N \backslash S} M_{k}(v)\right\}-\sum_{k \in S \backslash\{i\}} M_{k}(v) \\
& =\max \left\{m_{i}(v)+\sum_{k \in S \backslash\{i\}}\left(m_{k}(v)-M_{k}(v)\right), v(N)-\sum_{k \in N \backslash\{i\}} M_{k}(v)\right\} \\
& <m_{i}(v)
\end{aligned}
$$

where the first inequality follows from Theorem 2.1 and the second one is a direct consequence of $M(v)>m(v)$ and our supposition.

Second, let $m(v)=M(v)$. Since $v \in I^{N}$ is a compromise stable game and $m(v)=M(v)$, it follows that $\sum_{i \in N} m_{i}(v)=v(N)=\sum_{i \in N} M_{i}(v)$ and $n u c(v)=M(v)=\operatorname{AM}(N, E, c)=n u c^{1}(v)$.

Remark 5.1 As a consequence of Theorem 5.3, we can identify several well-known classes of compromise stable games for which the nucleolus and the 1-nucleolus coincide: big boss games (see Muto et al. 1988), clan games (see Potters et al. 1989), 1-convex games (see Driessen 1983) and 2-convex games (see Driessen 1983).

\section{Concluding remarks}

Similarly to the 1-nucleolus, one can consider $k$-nucleoli, with $k \in\{1, \ldots,|N|\}$, where only coalitions of size at most $k$ and at least $|N|-k$ are taken into account. Note that for ${ }^{2} k \geq\left\lfloor\frac{|N|}{2}\right\rfloor, n u c^{k}(v)=n u c(v)$ since all coalitions are considered. From a computational perspective, it could be interesting to further study the 2-nucleolus and to analyze explicit relationships between the 2-nucleolus and the nucleolus for special classes of games.

Another solution that selects an allocation on the basis of a restricted number of coalitional values is the Rawls rule (Tissdell and Harrison 1992), which considers only individual coalitions. The Rawls rule is also known as the centre of the imputation set (CIS-value, cf. Driessen and Funaki 1991) in the context of TU games. The 1nucleolus, in general, is different from the CIS-value. For instance, consider $N=$ $\{1,2,3\}, v(\{i\})=0$, for every $i \in N, v(\{1,2\})=4, v(\{1,3\})=0, v(\{2,3\})=3$, and $v(N)=6$. It turns out that $n u c^{1}(v)=(1.5,3.5,1)$, but $C I S(v)=(2,2,2)$.

An important line of research in TU-games is the axiomatic characterization of solutions based on desirable properties. As future research, it may be interesting to provide such a characterization of the 1-nucleolus for (special classes of) TU-games. In Table 1, we provide a comparative analysis of some properties regarding the 1nucleolus and the nucleolus for the class of balanced games. It is readily seen that both the 1-nucleolus and the nucleolus satisfy basic properties as invariance with respect to strategic equivalence, the dummy player property, and symmetry. It turns out that that the 1-nucleolus, contrary to the nucleolus, satisfies individual superadditivity

\footnotetext{
${ }^{2}$ For each $r \in \mathbb{R},\lfloor r\rfloor$ denotes the largest integer smaller than or equal to $r$.
} 
Table 1 Comparative analysis of the 1-nucleolus and the nucleolus by means of properties for the class of balanced games

\begin{tabular}{lll}
\hline Property & 1-nucleolus & Nucleolus \\
\hline Aggregate monotonicity & No & No \\
Individual superadditivity gains to the grand coalition & Yes & No \\
First agent consistency & Yes & No \\
\hline
\end{tabular}

gains to the grand coalition and first agent consistency (which is based on the idea of first-player consistency in Potters and Sudhölter (1999) and this, in turn, is based on Sobolev (1975)). Unfortunately, just like the nucleolus, the 1-nucleolus does not satisfy aggregate monotonicity either.

Let $f$ be a one point solution for TU-games. We say that $f$ satisfies

(i) aggregate monotonicity if for every balanced TU-games $(N, v)$ and $(N, w)$ satisfying $v(S)=w(S)$ for every $S \subseteq N, S \neq N$, and $w(N)>v(N)$, $f_{i}(N, w) \geq f_{i}(N, v)$ for every $i \in N$;

(ii) individual superadditivity gains to the grand coalition if for every balanced TUgame $(N, v)$ and every players $i, j \in N$ satisfying $v(N)-v(N \backslash\{i\})-v(\{i\}) \leq$ $v(N)-v(N \backslash\{j\})-v(\{j\}), f_{i}(N, v) \leq f_{j}(N, v) ;$

(iii) first agent consistency if for every balanced TU-game $(N, v)$ with $N=\{1, \ldots, n\}$ such that $v(N \backslash\{1\})+v(\{1\}) \geq v(N \backslash\{2\})+v(\{2\}) \geq \ldots \geq v(N \backslash\{n\})+v(\{n\})$ and such that $\left(N \backslash\{1\}, v_{1, f_{1}(N, v)}\right)$ is balanced, $f_{i}(N, v)=f_{i}\left(N \backslash\{1\}, v_{1, f_{1}(N, v)}\right)$ for every $i \in N \backslash\{1\}$; here, for $i \in N$ and $x \in \mathbb{R}$, the game $\left(N \backslash\{i\}, v_{i, x}\right)$ is defined by

$$
v_{i, x}(S)= \begin{cases}v(S) & \text { if } S \subseteq N \backslash\{i\},|S| \leq|N|-3 \\ v(S \cup\{i\})-x & \text { if } S \subseteq N \backslash\{i\},|N|-2 \leq|S| \leq|N|-1\end{cases}
$$

for every $S \subseteq N \backslash\{i\}$.

Acknowledgements The authors would like to thank an associate editor and two referees for their helpful suggestions to improve this article. Moreover, we would also like to thank the financial support of Ministerio de Ciencia e Innovación through Grant MTM2011-27731-C03 and Ministerio de Economía y Competitividad through Grant MTM2014-53395-C3-3-P.

Open Access This article is distributed under the terms of the Creative Commons Attribution 4.0 International License (http://creativecommons.org/licenses/by/4.0/), which permits unrestricted use, distribution, and reproduction in any medium, provided you give appropriate credit to the original author(s) and the source, provide a link to the Creative Commons license, and indicate if changes were made.

\section{Appendix: Proof of Theorem 4.1}

We assume, without loss of generality, that $N=\{1, \ldots, n\}$ and $c_{1} \leq c_{2} \leq \ldots \leq c_{n}$. Note that if $x \in I(v)$, then,

$$
e(\{j\}, x)=v(\{j\})-x_{j}=-x_{j}
$$


and

$$
e(N \backslash\{j\}, x)=v(N \backslash\{j\})-\sum_{k \in N \backslash\{j\}} x_{k}=v(N \backslash\{j\})-\left(v(N)-x_{j}\right)=x_{j}-c_{j}
$$

(i) We have $c_{j} \geq 0$ for every $j \in N$.

Case (i.a) $E \leq \sum_{j \in N} c_{j}$.

We show that $n u c^{1}(v)=\operatorname{AM}(N, E, c)$. Note that $(N, E, c)$ is a bankruptcy problem. We distinguish between two situations: $E \leq \frac{1}{2} \sum_{j \in N} c_{j}$ and $E>$ $\frac{1}{2} \sum_{j \in N} c_{j}$.

Case (i.a.1) $E \leq \frac{1}{2} \sum_{j \in N} c_{j}$.

By definition of the Aumann-Maschler rule, $\operatorname{AM}(N, E, c)=\operatorname{CEA}\left(N, E, \frac{1}{2} c\right)$ where $\operatorname{CEA}_{j}\left(N, E, \frac{1}{2} c\right)=\min \left\{\lambda, \frac{1}{2} c_{j}\right\}$ for every $j \in N$ and $\lambda \in \mathbb{R}_{+}$is chosen such that $\sum_{j \in N} \operatorname{CEA}_{j}\left(N, E, \frac{1}{2} c_{j}\right)=E$. Let $c_{0}=0$ and let $i \in N$ satisfy

$$
\frac{c_{i-1}}{2} \leq \lambda<\frac{c_{i}}{2} \text {. }
$$

Then, $\lambda=\frac{1}{|N|-i+1}\left(E-\sum_{k=0}^{i-1} \frac{c_{k}}{2}\right)$ and

$$
\operatorname{AM}_{j}(N, E, c)= \begin{cases}\frac{c_{j}}{2} & \text { if } 1 \leq j \leq i-1 \\ \lambda & \text { if } i \leq j \leq n\end{cases}
$$

Let $x=\operatorname{AM}(N, E, c)$. Then,

$$
e(\{j\}, x)=-x_{j}= \begin{cases}-\frac{c_{j}}{2} & \text { if } 1 \leq j \leq i-1 \\ -\lambda & \text { if } i \leq j \leq n\end{cases}
$$

and

$$
e(N \backslash\{j\}, x)=x_{j}-c_{j}= \begin{cases}-\frac{c_{j}}{2} & \text { if } 1 \leq j \leq i-1 \\ \lambda-c_{j} & \text { if } i \leq j \leq n\end{cases}
$$

Therefore,

$$
\begin{aligned}
e(\{1\}, x) & =e(N \backslash\{1\}, x) \geq \ldots \geq e(\{i-1\}, x) \\
& =e(N \backslash\{i-1\}, x)=-\frac{c_{i-1}}{2} \geq-\lambda \\
& =e(\{i\}, x)=\ldots=e(\{n\}, x)=-\lambda>-\lambda+2 \lambda-c_{i}=\lambda-c_{i} \\
& =e(N \backslash\{i\}, x) \geq \ldots \geq e(N \backslash\{n\}, x) .
\end{aligned}
$$

Let $i_{1}, i_{2}, \ldots, i_{p} \in\{1, \ldots, i-1\}$ be such that

$$
c_{1}=\ldots=c_{i_{1}}<c_{i_{1}+1}=\ldots=c_{i_{2}}<\ldots<c_{i_{p}+1}=\ldots=c_{i-1}<c_{i}
$$


and let $j_{1}, j_{2}, \ldots, j_{q} \in\{i, \ldots, n\}$ be such that

$$
c_{i}=\ldots=c_{j_{1}}<c_{j_{1}+1}=\ldots=c_{j_{2}}<\ldots<c_{j_{q}+1}=\ldots=c_{n} .
$$

Let $i_{0}=0, i_{p+1}=i-1, j_{0}=i-1$ and $j_{q+1}=n$. Using the same notation as in Theorem 3.3, we have $B_{0}^{1}(x, v)=\emptyset$ and

$B_{m}^{1}(x, v)= \begin{cases}\left\{\left\{i_{m-1}+1\right\}, N \backslash\left\{i_{m-1}+1\right\}, \ldots,\left\{i_{m}\right\}, N \backslash\left\{i_{m}\right\}\right\} & \text { if } 1 \leq m \leq p+1, \\ \{\{i\}, \ldots,\{n\}\} & \text { if } m=p+2, \\ \left\{N \backslash\left\{j_{m-p-3}+1\right\}, \ldots, N \backslash\left\{j_{m-p-2}\right\}\right\} & \text { if } p+3 \leq m \leq p+q+3\end{cases}$

Then, $\mathcal{B}^{1, l}(x, v)=\cup_{m=1}^{l} B_{m}^{1}(x, v)$ is balanced for every $l \in\{1,2, \ldots, p+q+3\}$ and, by Theorem 3.3, we have that $x=n u c^{1}(v)$.

Case (i.a.2) $E>\frac{1}{2} \sum_{j \in N} c_{j}$.

By definition of the Aumann-Maschler rule, $\operatorname{AM}(N, E, c)=c-$ CEA $\left(N, \sum_{k \in N} c_{k}-E, \frac{1}{2} c\right)$ where $\operatorname{CEA}_{j}\left(N, \sum_{k \in N} c_{k}-E, \frac{1}{2} c\right)=\min \left\{\lambda, \frac{1}{2} c_{j}\right\}$ for every $j \in N$ and $\lambda \in \mathbb{R}_{+}$is chosen such that $\sum_{j \in N} \operatorname{CEA}_{j}\left(N, \sum_{k \in N} c_{k}-E, \frac{1}{2} c\right)$ $=\sum_{k \in N} c_{k}-E$.

Let $c_{0}=0$ and let $i \in N$ satisfy

$$
\frac{c_{i-1}}{2} \leq \lambda<\frac{c_{i}}{2}
$$

Then, $\lambda=\frac{1}{|N|-i+1}\left(\sum_{k=1}^{|N|} c_{k}-E-\sum_{k=0}^{i-1} \frac{c_{k}}{2}\right)$ and

$$
\operatorname{AM}_{j}(N, E, c)= \begin{cases}\frac{c_{j}}{2} & \text { if } 1 \leq j \leq i-1 \\ c_{j}-\lambda & \text { if } i \leq j \leq n\end{cases}
$$

Let $x=\operatorname{AM}(N, E, c)$. Then,

$$
e(\{j\}, x)=-x_{j}= \begin{cases}-\frac{c_{j}}{2} & \text { if } 1 \leq j \leq i-1 \\ -c_{j}+\lambda & \text { if } i \leq j \leq n\end{cases}
$$

and

$$
e(N \backslash\{j\}, x)=x_{j}-c_{j}= \begin{cases}-\frac{c_{j}}{2} & \text { if } 1 \leq j \leq i-1, \\ -\lambda & \text { if } i \leq j \leq n\end{cases}
$$

Therefore,

$$
\begin{aligned}
e(\{1\}, x) & =e(N \backslash\{1\}, x) \geq \ldots \geq e(\{i-1\}, x)=e(N \backslash\{i-1\}, x) \\
& =-\frac{c_{i-1}}{2} \geq-\lambda \\
& =e(N \backslash\{i\}, x)=\ldots=e(N \backslash\{n\}, x)=-\lambda>-\lambda+2 \lambda-c_{i}=\lambda-c_{i} \\
& =e(\{i\}, x) \geq \ldots \geq e(\{n\}, x) .
\end{aligned}
$$


Then, similarly as in Case (i.a.1), we have that $x=n u c^{1}(v)$ by Theorem 3.3.

Case (i.b) $E>\sum_{j \in N} c_{j}$.

We show that $n u c^{1}(v)=c+\operatorname{ES}\left(N, E-\sum_{j \in N} c_{j}, c\right)$. Let $x=c+\operatorname{ES}(N, E-$ $\left.\sum_{j \in N} c_{j}, c\right)$. Then, $x_{j}=c_{j}+\frac{E-\sum_{k \in N} c_{k}}{|N|}$,

$$
\begin{aligned}
e(\{j\}, x) & =-x_{j}=-c_{j}-\frac{E-\sum_{k \in N} c_{k}}{|N|}, \quad \text { and } \\
e(N \backslash\{j\}, x) & =x_{j}-c_{j}=\frac{E-\sum_{k \in N} c_{k}}{|N|}
\end{aligned}
$$

for every $j \in N$. Therefore,

$$
e(N \backslash\{1\}, x)=\ldots=e(N \backslash\{n\}, x)>e(\{1\}, x) \geq \ldots \geq e(\{n\}, x)
$$

where the strict inequality is a direct consequence of the fact that $\frac{E-\sum_{k \in N} c_{k}}{|N|}>$ $0>-c_{1}-\frac{E-\sum_{k \in N} c_{k}}{|N|}$. Then, similarly as in Case (i.a.1), we have that $x=$ $n u c^{1}(v)$ by Theorem 3.3.

(ii) We have $c_{j}<0$ for some $j \in N$. Assume, without loss of generality, that $c_{1} \leq \ldots \leq c_{\bar{k}}<0 \leq c_{\bar{k}+1} \leq \ldots \leq c_{n}$ with $\bar{k} \in\{1, \ldots, n\}$.

Case (ii.a) $E \leq \sum_{j \in N} c_{j}^{+}$.

We show that $n u c^{1}(v)=\operatorname{AM}\left(N, E, c^{+}\right)$. Note that $c_{1}^{+}=\ldots=c_{\bar{k}}^{+}=0$ and $c_{j}^{+}=c_{j}$ for every $j \in\{\bar{k}+1, \ldots, n\}$. Moreover, $\left(N, E, c^{+}\right)$is a bankruptcy problem. By definition of the Aumann-Maschler rule, $\operatorname{AM}_{j}\left(N, E, c^{+}\right)=0$ for every $j \in\{1, \ldots, \bar{k}\}$. Let $x=\operatorname{AM}\left(N, E, c^{+}\right)$. Then,

$$
\begin{gathered}
e(\{j\}, x)=-x_{j}=0 \text { and } e(N \backslash\{j\}, x)=x_{j}-c_{j}=-c_{j}>0 \\
\text { for every } j \in\{1, \ldots, \bar{k}\} .
\end{gathered}
$$

Since $\operatorname{AM}_{j}\left(N, E, c^{+}\right)>0$ for every $j \in N$ with $c_{j}^{+}>0$, it follows that $B_{0}^{1}(x, v)=\{1, \ldots, \bar{k}\}$. Moreover, $\mathcal{B}_{1}^{1}(x, v)=\{N \backslash\{1\}, \ldots, N \backslash\{\bar{k}\}\}$ and $B_{0}^{1}(x, v) \cup \mathcal{B}_{1}^{1}(x, v)$ is balanced. Further, following the same lines as in Case (i.a.1) of this proof, one can see that $x=n u c^{1}(v)$.

Case (ii.b) $\sum_{j \in N} c_{j}^{+}<E \leq \sum_{j \in N} c_{j}^{\min }$.

We show that $n u c^{1}(v)=\operatorname{CEL}\left(N, E, c^{\text {min }}\right)$, with $\operatorname{CEL}_{j}\left(N, E, c^{\text {min }}\right)=$ $\max \left\{0, c_{j}^{\min }-\lambda\right\}$ for every $j \in N$ and $\lambda \in \mathbb{R}_{+}$chosen such that $\sum_{j \in N} \mathrm{CEL}_{j}$ $\left(N, E, c^{\min }\right)=E$. Note that $c_{j}^{\min }=c_{j}-\min \left\{c_{k} \mid k \in N\right\}=c_{j}-c_{1}$ for every $j \in N$ and $0=c_{1}^{\min } \leq c_{2}^{\min } \leq \ldots \leq c_{n}^{\min }$. Moreover, it follows that $\left(N, E, c^{\text {min }}\right)$ is a bankruptcy problem.

Let $i \in N$ satisfy

$$
c_{i-1}^{\min } \leq \lambda<c_{i}^{\min } .
$$


Then, $\lambda=\frac{1}{|N|-i+1}\left(\sum_{k=i}^{|N|} c_{k}^{\min }-E\right)$ and

$$
\mathrm{CEL}_{j}\left(N, E, c^{\min }\right)= \begin{cases}0 & \text { if } 1 \leq j \leq i-1 \\ c_{j}^{\min }-\lambda & \text { if } i \leq j \leq n\end{cases}
$$

Let $x=\operatorname{CEL}\left(N, E, c^{\min }\right)$. Then,

$$
e(\{j\}, x)=-x_{j}= \begin{cases}0 & \text { if } 1 \leq j \leq i-1 \\ -c_{j}^{\min }+\lambda & \text { if } i \leq j \leq n\end{cases}
$$

and

$$
e(N \backslash\{j\}, x)=x_{j}-c_{j}=x_{j}-c_{j}^{\min }-c_{1}= \begin{cases}-c_{j} & \text { if } 1 \leq j \leq i-1, \\ -c_{1}-\lambda & \text { if } i \leq j \leq n\end{cases}
$$

Before we write the excesses in non-increasing order, we show that

$$
i-1 \leq \bar{k}
$$

First, note that

$$
-c_{1}>\lambda
$$

since

$$
\begin{aligned}
\lambda & =\frac{1}{|N|-i+1}\left(\sum_{k=i}^{|N|} c_{k}^{\min }-E\right)=\frac{1}{|N|-i+1}\left(\sum_{k=i}^{|N|}\left(c_{k}-c_{1}\right)-E\right) \\
& =\frac{1}{|N|-i+1}\left(\sum_{k=i}^{|N|} c_{k}-E\right)-c_{1} \leq \frac{1}{|N|-i+1}\left(\sum_{k \in N} c_{k}^{+}-E\right)-c_{1}<-c_{1},
\end{aligned}
$$

where the weak inequality is a direct consequence of the definition of $c^{+}$and the strict inequality follows by the assumption $\sum_{k \in N} c_{k}^{+}<E$.

Next, we show that $i-1 \leq \bar{k}$ by contradiction. Suppose, on the contrary, that $i-1>\bar{k}$. Then, $c_{i-1}>0$ by definition of $\bar{k}$ and $c_{i-1}^{\min }=c_{i-1}-c_{1}>-c_{1}>$ $\lambda$. This establishes a contradiction with the definition of $i$. Therefore, we have $i-1 \leq \bar{k}$. Then,

$$
B_{0}^{1}(x, v)=\{1, \ldots, i-1\}
$$

and

$$
\begin{aligned}
e(N \backslash\{1\}, x) \geq \ldots & \geq e(N \backslash\{i-1\}, x) \geq e(N \backslash\{i\}, x)=\ldots=e(N \backslash\{n\}, x) \\
& >e(\{1\}, x)=\ldots=e(\{i-1\}, x)>e(\{i\}, x) \geq \ldots \geq e(\{n\}, x)
\end{aligned}
$$

where $e(N \backslash\{i-1\}, x) \geq e(N \backslash\{i\}, x)$ because $-c_{i-1}=-c_{1}-c_{i-1}^{\min } \geq-c_{1}-\lambda$ by definition of $i ; e(N \backslash\{n\}, x)>e(\{1\}, x)$ since $-c_{1}>\lambda$ and, then, $-c_{1}-\lambda>0$; 
$e(\{i-1\}, x)>e(\{i\}, x)$ since $c_{i}^{\min }>\lambda$ by definition of $i$. Then, similarly as in Case (i.a.1), we have that $x=n u c^{1}(v)$ by Theorem 3.3.

Case (ii.c) $E>\sum_{j \in N} c_{j}^{\min }$.

We show that $n u c^{1}(v)=c+\operatorname{ES}\left(N, E-\sum_{j \in N} c_{j}, c\right)$. Let $x=c+\operatorname{ES}(N, E-$ $\left.\sum_{j \in N} c_{j}, c\right)$. Then, $x_{j}=c_{j}+\frac{E-\sum_{k \in N} c_{k}}{|N|}$,

$$
\begin{gathered}
e(\{j\}, x)=-x_{j}=-c_{j}-\frac{E-\sum_{k \in N} c_{k}}{|N|}, \quad \text { and } \\
e(N \backslash\{j\}, x)=x_{j}-c_{j}=\frac{E-\sum_{k \in N} c_{k}}{|N|}
\end{gathered}
$$

for every $j \in N$. Therefore,

$$
e(N \backslash\{1\}, x)=\ldots=e(N \backslash\{n\}, x)>e(\{1\}, x) \geq \ldots \geq e(\{n\}, x)
$$

where the strict inequality is a direct consequence of the fact that $\frac{E-\sum_{k \in N} c_{k}}{|N|}>$ $0>-c_{1}-\frac{E-\sum_{k \in N} c_{k}}{|N|}$. Then, similarly as in Case (i.a.1), we have that $x=$ $n u c^{1}(v)$ by Theorem 3.3.

\section{Proofs or counterexamples to the properties on Table 1}

To see that the nucleolus and the 1-nucleolus do not satisfy aggregate monotonicity, we refer to the example in Tauman and Zapechelnyuk (2010), where both the nucleolus and 1-nucleolus coincide.

Since the 1-nucleolus of a balanced game $(N, v)$ is given by $n u c_{i}^{1}(v)=v(\{i\})+$ $\operatorname{AM}_{i}(N, E, c)$ for every $i \in N$, with $(N, E, c)$ the bankruptcy problem given by $E=v(N)-\sum_{j \in N} v(\{j\})$ and $c_{j}=v(N)-v(N \backslash\{j\})-v(\{j\})$ for every $j \in N$, it immediately follows that the 1-nucleolus satisfies individual superadditivity gains to the grand coalition since the Aumann-Maschler rule satisfies order preservation (cf. Thomson 2003). Example 5.2 shows that the nucleolus does not need to satisfy individual superadditivity gains to the grand coalition.

With respect to first agent consistency, Example 5.2 provides a balanced game for which the nucleolus does not satisfy first agent consistency. Next, we show that the 1-nucleolus satisfies first agent consistency. Let $(N, v)$ be a balanced TUgame with $N=\{1, \ldots, n\}$ such that $v(N \backslash\{1\})+v(\{1\}) \geq v(N \backslash\{2\})+v(\{2\}) \geq$ $\ldots \geq v(N \backslash\{n\})+v(\{n\})$ and such that $\left(N \backslash\{1\}, v_{1, f_{1}(N, v)}\right)$ is balanced. We show that $f_{i}(N, v)=f_{i}\left(N \backslash\{1\}, v_{1, f_{1}(N, v)}\right)$ for every $i \in N \backslash\{1\}$. We can assume, without loss of generality, that $v=v_{0}$. Then, by Theorem 4.2, $n u c^{1}(v)=\operatorname{AM}(N, E, c)$ with $E=v(N)$ and $c \in \mathbb{R}^{N}$ defined as $c_{j}=v(N)-v(N \backslash\{j\})$ for every $j \in N$. Note that $c_{1} \leq c_{2} \leq \ldots \leq c_{n}$. We distinguish between two cases: $E \leq \frac{1}{2} \sum_{j \in N} c_{j}$ and $E>\frac{1}{2} \sum_{j \in N} c_{j}$. 
Case $1 E \leq \frac{1}{2} \sum_{j \in N} c_{j}$ In this case,

$$
n u c^{1}(v)=\operatorname{CEA}\left(N, E, \frac{1}{2} c\right)
$$
by

and $n u c_{1}^{1}(v)=\min \left\{\frac{c_{1}}{2}, \frac{v(N)}{n}\right\}$. Besides, the game $\left(N \backslash\{1\}, v_{1, n u c_{1}^{1}(N, v)}\right)$ is given

$$
v_{1, n u c_{1}^{1}(N, v)}(S)= \begin{cases}v(N)-n u c_{1}^{1}(v) & \text { if } S=N \backslash\{1\}, \\ v(S \cup\{1\})-n u c_{1}^{1}(v) & \text { if }|S|=n-2, \\ v(S) & \text { otherwise. }\end{cases}
$$

Let $\tilde{E}=v(N)-n u c_{1}^{1}(v)$ and $\tilde{c} \in \mathbb{R}^{N \backslash\{1\}}$ with

$$
\tilde{c}_{j}=v_{1, n u c_{1}^{1}(N, v)}(N \backslash\{1\})-v_{1, n u c_{1}^{1}(N, v)}(N \backslash\{1, j\})=v(N)-v(N \backslash\{j\})=c_{j}
$$

for every $j \in N \backslash\{1\}$. First, if $n u c_{1}^{1}(v)=\frac{c_{1}}{2}$, then, $v(N)=E \leq \frac{1}{2} \sum_{j=1}^{n} c_{j}$ implies

$$
\tilde{E}=v(N)-\frac{c_{1}}{2} \leq \sum_{j=2}^{n} \frac{c_{j}}{2}=\sum_{j=2}^{n} \frac{\tilde{c}_{j}}{2}
$$

Second, if $n u c_{1}^{1}(v)=\frac{v(N)}{n}$, then, $\frac{c_{1}}{2} \leq \frac{v(N)}{n}$ and

$$
\tilde{E}=\frac{(n-1) v(N)}{n} \leq(n-1) \frac{c_{1}}{2} \leq \sum_{j=2}^{n} \frac{c_{j}}{2}=\sum_{j=2}^{n} \frac{\tilde{c}_{j}}{2}
$$

where the second inequality is a direct consequence of $c_{1} \leq c_{2} \leq \ldots \leq c_{n}$. Therefore,

$$
n u c^{1}\left(v_{1, n u c_{1}^{1}(N, v)}\right)=\operatorname{CEA}\left(N \backslash\{1\}, \tilde{E}, \frac{1}{2} c_{N \backslash\{1\}}\right)
$$

and

$$
\begin{aligned}
n u c_{2}^{1}\left(v_{1, n u c_{1}^{1}(N, v)}\right) & =\min \left\{\frac{c_{2}}{2}, \frac{v(N)-n u c_{1}^{1}(v)}{n-1}\right\} \\
& =\min \left\{\frac{c_{2}}{2}, \frac{v(N)-\operatorname{CEA}_{1}\left(N, E, \frac{1}{2} c\right)}{n-1}\right\} \\
& =\operatorname{CEA}_{2}\left(N, E, \frac{1}{2} c\right)=n u c_{2}^{1}(v) .
\end{aligned}
$$

Next, assume that $n u c_{j}^{1}\left(v_{1, n u c_{1}^{1}(N, v)}\right)=n u c_{j}^{1}(v)$ for every $j=2, \ldots, i-1$, with $i \in N \backslash\{1,2\}$. We show that $n u c_{i}^{1}\left(v_{1, n u c_{1}^{1}(N, v)}\right)=n u c_{i}^{1}(N, v)$. Note that 
$\mathrm{CEA}_{j}\left(N, \tilde{E}, \frac{1}{2} c\right)=n u c_{j}^{1}\left(v_{1, n u c_{1}^{1}(N, v)}\right)=n u c_{j}^{1}(v)=\mathrm{CEA}_{j}\left(N, E, \frac{1}{2} c\right)$ for $j=$ $2, \ldots, i-1$ and

$$
\begin{aligned}
n u c_{i}^{1}\left(v_{1, n u c_{1}^{1}(N, v)}\right) & =\min \left\{\frac{c_{j}}{2}, \frac{\tilde{E}-\sum_{j=2}^{i-1} \operatorname{CEA}_{j}\left(N, \tilde{E}, \frac{1}{2} c\right)}{n-i+1}\right\} \\
& =\min \left\{\frac{c_{j}}{2}, \frac{v(N)-n u c_{1}^{1}(v)-\sum_{j=2}^{i-1} \mathrm{CEA}_{j}\left(N, \tilde{E}, \frac{1}{2} c\right)}{n-i+1}\right\} \\
& =\min \left\{\frac{c_{j}}{2}, \frac{v(N)-\sum_{j=1}^{i-1} \mathrm{CEA}_{j}\left(N, E, \frac{1}{2} c\right)}{n-i+1}\right\} \\
& =\operatorname{CEA}_{i}\left(N, E, \frac{1}{2} c\right)=n u c_{i}^{1}(v) .
\end{aligned}
$$

Case $2 E>\frac{1}{2} \sum_{j \in N} c_{j}$

In this case,

$$
n u c^{1}(v)=c-\mathrm{CEA}\left(N, \sum_{j \in N} c_{j}-E, \frac{1}{2} c\right)
$$

$n u c_{1}^{1}(v)=c_{1}-\min \left\{\frac{c_{1}}{2}, \frac{\sum_{j=1}^{n} c_{j}-v(N)}{n}\right\}$. Besides, the game $\left(N \backslash\{1\}, v_{1, n u c_{1}^{1}(N, v)}\right)$ is given by

$$
v_{1, n u c_{1}^{1}(N, v)}(S)= \begin{cases}v(N)-n u c_{1}^{1}(v) & \text { if } S=N \backslash\{1\}, \\ v(S \cup\{1\})-n u c_{1}^{1}(v) & \text { if }|S|=n-2, \\ v(S) & \text { otherwise. }\end{cases}
$$

Let $\tilde{E}=v(N)-n u c_{1}^{1}(v)$ and $\tilde{c} \in \mathbb{R}^{N \backslash\{1\}}$ with

$$
\tilde{c}_{j}=v_{1, n u c_{1}^{1}(N, v)}(N \backslash\{1\})-v_{1, n u c_{1}^{1}(N, v)}(N \backslash\{1, j\})=c_{j}
$$

for every $j \in N \backslash\{1\}$. First, if $n u c_{1}^{1}(v)=\frac{c_{1}}{2}, v(N)>\frac{1}{2} \sum_{j=1}^{n} \frac{c_{j}}{2}$ implies

$$
\tilde{E}=v(N)-\frac{c_{1}}{2}>\frac{1}{2} \sum_{j=2}^{n} c_{j}=\frac{1}{2} \sum_{j=2}^{n} \tilde{c}_{j}
$$


Second, if $n u c_{1}^{1}(v)=c_{1}-\frac{\sum_{j=1}^{n} c_{j}-v(N)}{n}$, then,

$$
\begin{aligned}
\tilde{E} & =v(N)-c_{1}+\frac{\sum_{j=1}^{n} c_{j}-v(N)}{n}=\sum_{j=2}^{n} c_{j}-\frac{n-1}{n}\left(\sum_{j=1}^{n} c_{j}-v(N)\right) \\
& =\sum_{j=2}^{n}\left(c_{j}-\frac{\sum_{k=1}^{n} c_{k}-v(N)}{n}\right) \geq \sum_{j=2}^{n} \frac{c_{j}}{2}=\frac{1}{2} \sum_{j=2}^{n} \tilde{c}_{j} .
\end{aligned}
$$

where the inequality is a direct consequence of $c_{j}-\frac{\sum_{k=1}^{n} c_{k}-v(N)}{n} \geq \frac{c_{j}}{2}$ for every $j \in$ $\{2, \ldots, n\}$. To see this, note that $c_{1}-\frac{\sum_{j=1}^{n} c_{j}-v(N)}{n} \geq \frac{c_{1}}{2}$ implies $\frac{c_{1}}{2}-\frac{\sum_{j=1}^{n} c_{j}-v(N)}{n} \geq$ 0 . Then, since $c_{j} \geq c_{1}$ for $j \in\{2, \ldots, n\}$, we have $\frac{c_{j}}{2}-\frac{\sum_{k=1}^{n} c_{k}-v(N)}{n} \geq \frac{c_{1}}{2}-$ $\frac{\sum_{k=1}^{n} c_{k}-v(N)}{n} \geq 0$ and $c_{j}-\frac{\sum_{k=1}^{n} c_{k}-v(N)}{n} \geq \frac{c_{j}}{2}$ for every $j \in\{2, \ldots, n\}$. Therefore,

$$
n u c^{1}\left(v_{1, n u c_{1}^{1}(N, v)}\right)=c-\mathrm{CEA}\left(N \backslash\{1\}, \sum_{j=2}^{n} c_{j}-\tilde{E}, \frac{1}{2} c_{N \backslash\{1\}}\right)
$$

and

$$
\begin{aligned}
n u c_{2}^{1}\left(v_{1, n u c_{1}^{1}(N, v)}\right) & =c_{2}-\mathrm{CEA}_{2}\left(N \backslash\{1\}, \sum_{j=2}^{n} c_{j}-\tilde{E}, \frac{1}{2} c_{N \backslash\{1\}}\right) \\
& =c_{2}-\min \left\{\frac{c_{2}}{2}, \frac{\left.\sum_{j=2}^{n} c_{j}-\tilde{E}\right\}}{n-1}\right\} \\
& =c_{2}-\min \left\{\frac{c_{2}}{2}, \frac{\sum_{j=2}^{n} c_{j}-\left(v(N)-n u c_{1}^{1}(v)\right)}{n-1}\right\} \\
& =c_{2}-\min \left\{\frac{c_{2}}{2}, \frac{\sum_{j=1}^{n} c_{j}-v(N)-\left(c_{1}-n u c_{1}^{1}(v)\right)}{n-1}\right\} \\
& =c_{2}-\min \left\{\frac{c_{2}}{2}, \frac{\sum_{j=1}^{n} c_{j}-E-\min \left\{\frac{c_{1}}{2}, \frac{\sum_{j=1}^{n} c_{j}-E}{n}\right\}}{n-1}\right\} \\
& =c_{2}-\min \left\{\frac{c_{2}}{2}, \frac{\sum_{j=1}^{n} c_{j}-E-\mathrm{CEA}_{1}\left(N, \sum_{j=1}^{n} c_{j}-E, \frac{1}{2} c\right)}{n-1}\right\} \\
& =c_{2}-\operatorname{CEA}_{2}\left(N, \sum_{j=1}^{n} c_{j}-E, \frac{1}{2} c\right)=n u c_{2}^{1}(v) .
\end{aligned}
$$

Following the same lines as in the case above, one can see that $n u c_{j}^{1}\left(v_{1, n u c_{1}^{1}(N, v)}\right)=$ $n u c_{j}^{1}(N, v)$ for $j=2, \ldots, n$. 


\section{References}

Aumann R, Maschler M (1985) Game theoretic analysis of a bankruptcy problem from the Talmud. J Econ Theory 36:195-213

Bondareva ON (1963) Some applications of linear programming methods to the theory of cooperative games. Problemy Kibernitiki 10:119-139 (in Russian)

Driessen TSH (1983) Cores of $k$-convex $n$-person games with an increasing gap function. Report 8348. Department of Mathematics, Catholic University, Nijmegen

Driessen TSH, Funaki Y (1991) Coincidence of and collinearity between game theoretic solutions. OR Spektrum 13:15-30

Gillies DB (1953) Some theorems on $n$-person games. PhD thesis, Princeton University

Kohlberg E (1971) On the nucleolus of characteristic function game. SIAM J Appl Math 20:62-66

Kopelowitz A (1967) Computation of the kernels of simple games and nucleolus of $N$-person games. No. RM-31. Hebrew University Jerusalem (Israel) Department of Mathematics

Maschler M, Peleg B, Shapley LS (1979) Geometric properties of the kernel, nucleolus and related solution concepts. Math Oper Res 4:303-338

Maschler M, Potters J, Tijs SH (1992) The general nucleolus and the reduced game property. Int J Game Theory 21:85-106

Muto S, Nakayama M, Potters J, Tijs SH (1988) On big boss games. Econ Stud Q 39:303-321

O’Neill B (1982) A problem of rights arbitration from the Talmud. Math Soc Sci 2:345-371

Potters J, Poos R, Muto S, Tijs SH (1989) Clan games. Games Econ Behav 1:275-293

Potters J, Sudhölter P (1999) Airport problems and consistent allocation rules. Math Soc Sci 38:83-102

Quant M, Borm P, Reijnierse H, van Velzen B (2005) The core cover in relation to the nucleolus and the Weber set. Int J Game Theory 33:491-503

Sánchez-Rodríguez E, Borm P, Estévez-Fernández A, Fiestras-Janeiro MG, Mosquera MA (2015) $k$-core covers and the core. Math Methods Oper Res 81:147-167

Schmeidler D (1969) The nucleolus of a characteristic function game. SIAM J Appl Math 17:1163-1170

Schmeidler D (1972) Cores of exact games. J Math Anal Appl 40:214-225

Shapley LS (1953) A Value for $n$-person Games. In: Kuhn HW, Tucker AW (eds) Contributions to the theory of games, volume II, annals of mathematical studies vol 28, pp 307-317

Shapley LS (1967) On balanced sets and cores. Nav Res Logist Q 14:453-460

Shapley LS (1971) Cores of convex games. Int J Game Theory 1:11-26

Shapley LS, Shubik M (1972) The assignment game I: the core. Int J Game Theory 1:111-130

Sobolev AI (1975) The characterization of optimality principles in cooperative games by functional equations. In: Vorobiev NN (ed) Mathematical methods in social sciences 6. Academy of Sciences of the Lithuanian SSR, Vilnius, pp 95-151 (in Russian)

Tauman Y, Zapechelnyuk A (2010) On (non-)monotonicity of cooperative solutions. Int J Game Theory 39:171-175

Tijs SH (1981) Bounds for the core and the $\tau$-value. In: Pallaschke D, Moeschlin O (eds) Game theory and mathematical economics. North Holland Publishing Company, Amsterdam, pp 123-132

Tijs SH, Lipperts F (1982) The hypercube and the core cover of the $n$-person cooperative games. Cahiers du Centre d'Études de Recherche Opérationnelle 24:27-37

Thomson W (2003) Axiomatic and game-theoretic analysis of bankruptcy and taxation problems: a survey. Math Soc Sci 45:249-297

Tissdell JG, Harrison SR (1992) Estimating an optimal distribution of water entitlements. Water Resour Res 28:3111-3117 